\title{
Medicines for children in Europe: from proposals to practicalities
}

\section{To the Editors:}

It is widely recognised that sick children are being offered a second-rate service, because in many cases medications being used in critically ill young people have not been tested at all in the very constituency they are meant to help [1]. The many practical difficulties of clinical trials work in children have been reviewed, and welcome initiatives by the European Union (EU) among others have been put in place to try to address this. We now need to ensure that this leads to genuinely improved services for children. The two important questions are, what needs to be done, in order of priority; and how should our aims be achieved? The European Respiratory Society (ERS) has already been very active in both areas.

Clearly prioritisation is important; although we would like to inform all our decisions with good-quality randomised controlled trials in all the illnesses suffered by children at every age, in practice the scarcity of patients and the cost of trials means this will not happen for every treatment for each paediatric respiratory disease, at least in the short to medium term. Prioritisation needs to be in an open manner, free from the vested interests of the pharmaceutical industry, and with clear declarations of conflicts of interest from those debating the issues. The obvious structure to achieve this is through ERS Task Forces, and to that end, a review of the evidence in preschool children with wheezing disorders has been published [2]. Two further Task Forces, on the evidence (or lack of it) for the use of medications for respiratory issues found only in children, and another on respiratory problems found in children and adults, have been published more recently [3, 4]. In all cases, the focus is on what is known from studies in children, as distinct from the much less satisfactory practice of extrapolation from studies in adults. It is hoped that these three reviews will provide the objective evidence base needed to inform the deliberations of those who will need to invest the money to carry out these studies.

For most paediatric diseases, the diversity, complexity and agerelated differences will mean that the role of the single-centre study becomes much smaller. Already there have been welcome initiatives; there are well-developed networks in a number of European countries, for example the Medicines for Children network in the UK. The European Academy of Paediatrics (EAP), as the European umbrella organisation for paediatrics that also serves as the paediatric section of the Union of European Medical Specialists has also been particularly active in supporting the new EU legislation on medicines for children. Its support ranged from lobbying in the European Parliament, to raising awareness through various meetings, statements and presentations. Taking the relevant US legislation and its obvious success as a guideline and stimulus, it thereby strongly supported European Medicines Evaluation Agency (EMEA) in its search for these urgently needed new regulations. Now, with this legislation having been in place for over a year, it becomes evident that its effects meet or even exceed expectations. Patent extension as a bonus for clinical trials in children proves to be a strong stimulus for an early and careful exploration of pharmacokinetics, therapeutic effects and side-effect risks in the paediatric population. The resulting oncoming wave of clinical trials in children will ensure a safer, evidence-based and child-specific prescription of new substances in the future. As a side-effect of this development, however, there will be a rising need for highly competent clinical trial networks in the various areas and subspecialities of paediatrics. Among others, paediatric respiratory medicine will certainly be challenged to provide competent trial networks for evaluating new medications for asthma, cystic fibrosis (CF) and other respiratory disorders. The EAP, via its established relations to EMEA, offers itself as a link between pharmaceutical industry and EMEA on one side, and interested clinical centres on the other. Together with various paediatric subspeciality societies, it intends to serve as a guarantor of quality on the way from the development of a promising new substance towards the necessary spectrum of meaningful clinical study results. As a pilot project in this field, the EAP has suggested to the ERS Paediatrics Assembly that a list of trial centres competent in the paediatric respiratory field should be compiled. This idea was endorsed and executed by the ERS Paediatrics Assembly. The resulting list has already raised considerable interest within EMEA and the pharmaceutical industry and among some members of the European Parliament. It will serve as pilot project example for other interested paediatric subspecialities.

This list of nearly 100 centres that have declared an interest is now available on the ERS website [5]. It is important to appreciate the strengths and weaknesses of this list. The strength is that it is probably the biggest international group that has expressed an interest in paediatric clinical trials work. It is, however, a self-assessment both of capacity and the techniques and competencies available, and all users of the list need to be aware of this. There has been to date no outside objective verification of the claims of any centre by the ERS. One of the lessons of the enormously successful US CF Foundation Therapeutic Drugs Network is that great efforts need to be made to ensure standardisation of all techniques used in multiple centres, and standardisation cannot be assured in the list in its current form, although there is no reason why this cannot be achieved in the future.

How then should this list be used by those organising clinical trials in children? It is a resource of centres that may be interested, and thus would be happy to receive proposals. The principal investigator has to convince the relevant centres that the proposal is important, doable, attractive and adequately resourced. The centre has to persuade the investigator that they can recruit from a pool of the right patients, and can perform the techniques needed appropriate standards. The list cannot be a substitute for site visits, which are a prerequisite for properly controlled, multicentre trials of any sophistication. 
Where then are we going, and how far have we got? It is clear that new initiatives in medicines for children represent a oncein-a-career opportunity. We need the reality of truly evidencebased therapy for children, not merely in common conditions, but also in rarities. The hope is that the current ERS and EAP initiatives will help to move this important field forward in a good way for children.

\section{A. Bush* and M. Zach ${ }^{\#}$}

*Imperial College and Royal Brompton Hospital, London, UK. \#Medical University of Graz, Graz, Austria.

Correspondence: A. Bush, Dept of Paediatric Respiratory Medicine, Royal Brompton Hospital, Sydney Street, London SW3 6NP, UK. E-mail: a.bush@rbh.nthames.nhs.uk

\section{REFERENCES}

1 Bush A. Evidence-based medicines for children: important implications for new therapies at all ages. Eur Respir J 2006; 28: 1069-1072.

2 Brand P, Baraldi E, Bisgaard H, et al. Definition, assessment and treatment of wheezing disorders in preschool children: an evidencebased approach. Eur Respir J 2008; 32: 1096-1110.

3 Lenney W, Boner AZ, Bont L, et al. Medicines used in respiratory diseases only seen in children. Eur Respir J 2009; 34: 531-551.

4 Smyth A, Barbato A, Beydon N, et al. Respiratory medicines for children: current evidence, unlicensed use, and research priorities. Eur Respir J 2009 [Epub ahead of print DOI: 10.1183/09031936. 00139508].

5 European Respiratory Society Paediatrics Assembly. List of centres who have expressed an interest in doing clinical trials. http://dev. ersnet.org/uploads/Document/b8/WEB_CHEMIN_3221_1215089384. doc Date last accessed: May 21, 2009.

Statement of Interest: None declared. 